\title{
A New Understanding of Equivalence of the Accelerating Field and the Gravitational Field
}

\author{
Jidong Zhu \\ Shanghai University of Electric Power, Shanghai, China \\ Email: zhu_jidong@126.com
}

How to cite this paper: Zhu, J.D. (2016) A New Understanding of Equivalence of the Accelerating Field and the Gravitational Field. Open Access Library Journal, 3: e3034. http://dx.doi.org/10.4236/oalib.1103034

Received: September 4, 2016

Accepted: September 26, 2016

Published: September 30, 2016

Copyright $\odot 2016$ by author and Open Access Library Inc.

This work is licensed under the Creative Commons Attribution International License (CC BY 4.0).

http://creativecommons.org/licenses/by/4.0/

(c) (i) Open Access

\begin{abstract}
According to the equivalence principle, the accelerating field and the gravitational field are considered to be completely equivalent. But after carefully studying the two most classic acceleration systems, i.e. the uniform acceleration elevator and the uniform rotating disc, the author has discovered that there is only partial equivalence instead of complete equivalence between the above two acceleration systems and the gravitational field.
\end{abstract}

\section{Subject Areas}

Classical Physics

\section{Keywords}

Equivalence Principle, Gravitational Field, Accelerating Field, Bending of Light

\section{Preface}

In recent decades, the study of relativity gradually is gradually becoming active. It is generally known that just like Newtonian Mechanics has its limitation, the relativity also has its limitation. So far, there is no experimental basis for the Relativistic space-time outlook, and the author's study shows that problems exist in the Equivalence principle. When proving equivalence principle, Einstein just used several ideal experiments. The uniform acceleration elevator and the uniform rotating disc are the two most classic acceleration systems among the several ideal experiments. After careful analysis, the author discovered that the two most classic acceleration systems and the gravitational field are not completely equivalent. 


\section{The Uniform Acceleration Elevator and the Gravitational Field Is Not Completely Equivalent}

\subsection{Light Bending in Gravitational Field}

According to the General Theory of Relativity, the light spreads out by a straight line in a vacuum without gravity, but it will bend in the gravitational field. Figure 1(a) is a schematic diagram of an elevator, let's assume that the width of the elevator is $3 \mathrm{~m}$. If the elevator is stationary in a vacuum without gravity, and the outside light gets in from the point $A_{0}$ along the direction parallel to the floor of the elevator, it will reach the point $B_{0}$ on the opposite wall of the elevator. In Figure 1(a), the author draws out a virtual line $A_{0} B_{0}$, which indicates the linear propagation of the light in a vacuum. But if the elevator is stationary on the surface of a planet and the direction of gravity of the planet is shown in the arrow in the figure, the path of propagation of light in the gravitational field is curved. The outside light which gets in from the point $A_{0}$ will reach the point $B_{1}$ on the opposite wall of the elevator, the position of the point $B_{1}$ is a little lower than the point $B_{0}$. In Figure 1 (a), the author also draws out a curve $\hat{A}_{0} \hat{B}_{1}$, which indicates that the light is bending in the gravitational field.

Theoretically, when the elevator is parked on the surface of the planet, the trajectory of the photon in the elevator should be a curve, but the width of the elevator is only 3 meters, in such a short distance when we calculate the deflection angle of the light, we can use a slash instead of a curve, and the result will remain unchanged. For this reason, in Figure $1(\mathrm{~b})$, point $A_{0}$, point $B_{1}$ were connected by a solid line, the line $A_{0} B_{1}$ replaced the curve $\hat{A}_{0} \hat{B}_{1}$. The angle $\alpha$ between solid line $A_{0} B_{1}$ and dashed lines $A_{0} B_{0}$ can be regarded as the deflection angle of light.

When the elevator is parked on the surface of the planet, we suppose that from point $A_{0}$, the 1st light pulse gets in at time $t_{1}$, the 2nd light pulse gets in at time $t_{2}, \ldots$, the $n$th light pulse gets in at time $t_{n}$, the observer inside the elevator will find that the trajectories of the light pulses are consistent. That is to say, if every light pulse gets in form point $A_{0}$, they will get to the same point $B_{1}$ on the opposite wall of the elevator. Their deflection angles of light are the same.

Where the gravitational force is greater and the gravitational potential is deeper, the bending of the light is more powerful. The General Theory of Relativity predicts that the photon will be deflected at the edge of the sun, and deflection angle is:

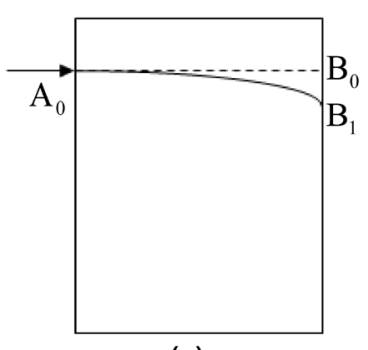

(a)

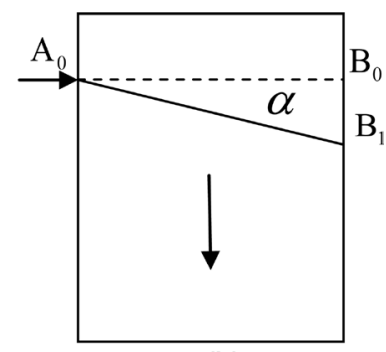

(b)

Figure 1. (a) The bending light in the gravitational field; (b) the deflection angle of light in the gravitational field. gravitational field. 


$$
\tan \phi \approx \phi \approx \frac{4 G M_{s}}{R_{s} c^{2}} \approx 1.75^{\prime \prime}
$$

In the above formula, $M_{s}$ is the mass of the sun, $R_{s}$ is the radius of the sun. Experiments have already proved that the prediction given by the General Theory of Relativity is correct. Light will also deflect when passing through the earth's surface. Use $g_{e}$ to represent the earth's gravitational field, and use $g_{s}$ to represent the gravitational field at the edge of the sun. Because the earth's mass is far less than the sun's mass, so $g_{e} \ll g_{s}$, the deflection of the light is negligible and can be ignored in the earth's reference frame.

\subsection{Light Bending in Uniform Acceleration Elevator}

Einstein believed that the gravitational field and the accelerating field are completely equivalent, so the light will also bend in the uniform acceleration rising elevator [1]. Figure 2 is also a schematic diagram of an elevator. The width $w$ of the elevator is also $3 \mathrm{~m}$, and the light gets in from the point $A_{0}^{\prime}$. When the elevator is stationary, the light travels in straight lines reaching the point $B_{0}^{\prime}$ on the opposite wall of the elevator. In Figure 2, the author draws out a virtual line $A_{0}^{\prime} B_{0}^{\prime}$ to represent the rectilinear propagation of the light. The time the light requires from point $A_{0}^{\prime}$ to get to point $B_{0}^{\prime}$ is:

$$
\Delta t^{\prime}=\frac{w}{c}=\frac{3}{3 \times 10^{8}}=1 \times 10^{-8} s=10 \mathrm{~ns}
$$

\subsubsection{Estimation of Deflection Angle of Light in the Uniform Acceleration Elevator}

The elevator in Figure 2 starts to do uniform acceleration movement at time $t_{0}^{\prime}$, with the accelerated speed $a$ :

$$
a=g_{e} \approx 10 \mathrm{~m} / \mathrm{s}^{2}
$$

The direction of the arrow in the Figure 2 is the direction of the elevator acceleration. Suppose the initial velocity of the elevator is zero. In the gravitational field of the earth, the deflection effect of the light can be ignored, however, the following analysis shows that even in the case of $a=g_{e}$, the trajectories of light in the uniform acceleration elevator and the trajectories of the light in the earth's Gravitational field are not the same, the deflection effect of the light can not be ignored in the uniform acceleration elevator.

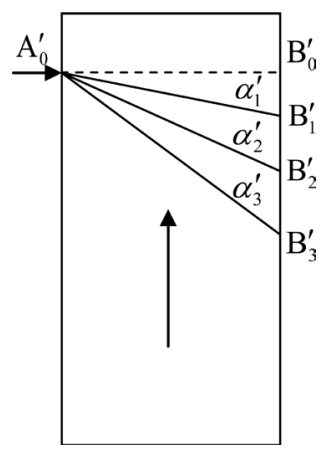

Figure 2. The deflection angle of light in the acceleration elevator. 
Einstein pointed out that in uniform acceleration elevator the propagation path of the light is curved. The observer inside the elevator may think that the bending of light is caused by gravity, and for the observer outside the elevator, the light still travels in a straight line. But because of acceleration movement of the elevator, in this period of time when the photons from the incident point reach the opposite wall, the position of the elevator has changed, so the illumination point of the light on the opposite wall is slightly lower than the point $B_{0}^{\prime}[1]$. According to Einstein's ideas, let's estimate the deflection angle of the light in the uniform acceleration elevator.

Assuming that there are three external light pulses along the direction parallel to the floor of the elevator, orderly get in from the point $A_{0}^{\prime}$ of the elevator, and after $10 \mathrm{~ns}$ get to the opposite wall. The 1st light pulse gets in at time $t_{0}^{\prime}$ and gets to the point $B_{1}^{\prime}$ of the opposite wall at time $t_{1}^{\prime}$. The 2 nd light pulse gets in at time $t_{1}^{\prime}$ and gets to the point $B_{2}^{\prime}$ of the opposite wall at time $t_{2}^{\prime}$. The 3 rd light pulse gets in at time $t_{2}^{\prime}$ and gets to the point $B_{3}^{\prime}$ of the opposite wall at time $t_{3}^{\prime}$. Obviously the following relationship is established:

$$
t_{1}^{\prime}-t_{0}^{\prime}=t_{2}^{\prime}-t_{1}^{\prime}=t_{3}^{\prime}-t_{2}^{\prime}=\cdots=t_{n}^{\prime}-t_{n-1}^{\prime}=10 \mathrm{~ns}
$$

For ease of calculation, set $t_{0}^{\prime}=0$, then the distance between the point $B_{1}^{\prime}$ and the point $B_{0}^{\prime}$ can be obtained as below:

$$
B_{0}^{\prime} B_{1}^{\prime}=\int_{t_{0}^{\prime}}^{t_{1}^{\prime}} v \mathrm{~d} t=a \int_{t_{0}^{\prime}}^{t_{1}^{\prime}} \mathrm{d} t=\frac{a\left(\left(t_{1}^{\prime}\right)^{2}-\left(t_{0}^{\prime}\right)^{2}\right)}{2}=\frac{a\left(\left(1^{2}-0^{2}\right) \times 10^{-16}\right)}{2}=0.5 \times 10^{-15} \mathrm{~m}
$$

In the same way, the distance between the point $B_{2}^{\prime}$ and the point $B_{0}^{\prime}$ can be obtained as below:

$$
B_{0}^{\prime} B_{2}^{\prime}=\int_{t_{1}^{\prime}}^{t_{2}^{\prime}} v \mathrm{~d} t=a \int_{t_{1}^{\prime}}^{t_{2}^{\prime}} t d t=\frac{a\left(\left(t_{2}^{\prime}\right)^{2}-\left(t_{1}^{\prime}\right)^{2}\right)}{2}=\frac{a\left(\left(2^{2}-1^{2}\right) \times 10^{-16}\right)}{2}=1.5 \times 10^{-15} \mathrm{~m}
$$

And the distance between the point $B_{3}^{\prime}$ and the point $B_{0}^{\prime}$ can be obtained as below:

$$
B_{0}^{\prime} B_{3}^{\prime}=\int_{t_{2}^{\prime}}^{t_{3}^{\prime}} v \mathrm{~d} t=a \int_{t_{2}^{\prime}}^{t_{3}^{\prime}} t d t=\frac{a\left(\left(t_{3}^{\prime}\right)^{2}-\left(t_{2}^{\prime}\right)^{2}\right)}{2}=\frac{a\left(\left(3^{2}-2^{2}\right) \times 10^{-16}\right)}{2}=2.5 \times 10^{-15} \mathrm{~m}
$$

In the General Theory of Relativity, the bending of the light reflects the bending of the space. If the space-time relationship between the accelerating system and the gravitational field is exactly equivalent, the observers in the acceleration elevator will also find that the trajectories of the three light beams from outside are completely coincident, and the three points $B_{1}^{\prime}, B_{2}^{\prime}$ and $B_{3}^{\prime}$ are at the same position. But the formulas (2)-(4) tell us that the points $B_{1}^{\prime}, B_{2}^{\prime}$ and $B_{3}^{\prime}$ are not at the same position. Now let's calculate the deflection angle of the light in the elevator, because the deflection angle is very small, so $\tan \alpha_{1} \approx \alpha_{1}$, the deflection angle of the 1 st beam is:

$$
\tan \alpha_{1} \approx \alpha_{1} \approx \frac{B_{0}^{\prime} B_{1}^{\prime}}{W}=\frac{a\left(\left(t_{1}^{\prime}\right)^{2}-\left(t_{0}^{\prime}\right)^{2}\right)}{2 c\left(t_{1}^{\prime}-t_{0}^{\prime}\right)}=\frac{a\left(t_{1}^{\prime}+t_{0}^{\prime}\right)}{2 c} \approx \frac{1 \times 10^{-7}}{6 \times 10^{8}} \approx 1.667 \times 10^{-16} \mathrm{rad}
$$


The deflection angle of the 2 nd beam is:

$$
\tan \alpha_{2} \approx \alpha_{2} \approx \frac{B_{0}^{\prime} B_{2}^{\prime}}{W}=\frac{a\left(\left(t_{2}^{\prime}\right)^{2}-\left(t_{1}^{\prime}\right)^{2}\right)}{2 c\left(t_{2}^{\prime}-t_{1}^{\prime}\right)}=\frac{a\left(t_{2}^{\prime}+t_{1}^{\prime}\right)}{2 c} \approx \frac{3 \times 10^{-7}}{6 \times 10^{8}} \approx 5 \times 10^{-16} \mathrm{rad}
$$

The deflection angle of the $3 \mathrm{rd}$ beam is:

$$
\tan \alpha_{3} \approx \alpha_{3} \approx \frac{B_{0}^{\prime} B_{3}^{\prime}}{W}=\frac{a\left(\left(t_{3}^{\prime}\right)^{2}-\left(t_{2}^{\prime}\right)^{2}\right)}{2 c\left(t_{3}^{\prime}-t_{2}^{\prime}\right)}=\frac{a\left(t_{3}^{\prime}+t_{2}^{\prime}\right)}{2 c} \approx \frac{5 \times 10^{-7}}{6 \times 10^{8}} \approx 8.33 \times 10^{-16} \mathrm{rad}
$$

$\cdots, \cdots$

The deflection angle of the $n$th beam is:

$$
\tan \alpha_{n} \approx \alpha_{n} \approx \frac{B_{0}^{\prime} B_{n}^{\prime}}{W}=\frac{a\left(\left(t_{n}^{\prime}\right)^{2}-\left(t_{n-1}^{\prime}\right)^{2}\right)}{2 c\left(t_{n}^{\prime}-t_{n-1}^{\prime}\right)}=\frac{a\left(t_{n}^{\prime}+t_{n-1}^{\prime}\right)}{2 c}
$$

In the case of $n \gg 1, t_{n}^{\prime} \approx t_{n-1}^{\prime}$, so

$$
\tan \alpha_{n} \approx \alpha_{n} \approx \frac{a\left(t_{n}^{\prime}+t_{n-1}^{\prime}\right)}{2 c} \approx \frac{2 a t_{n}^{\prime}}{2 c}=\frac{a t_{n}^{\prime}}{c}
$$

In formula (9), $v_{n}^{\prime}$ is the instantaneous speed of the elevator at $t_{n}^{\prime}$ time. According to formula (9), as time goes on, the speed $v_{n}^{\prime}$ will be faster and faster, the deflection angle $\alpha_{n}$ of the light will be bigger and bigger in the uniform acceleration elevator,.

Comparing Figure 1(a) and Figure 2, you could find that the light propagation characteristics in the uniform acceleration elevator and the light propagation characteristics in the gravitational field are not the same. When the elevator is parked on the surface of the planet, each light pulse from the point $A_{0}$ of the elevator always gets to the point $B_{1}$ on the opposite wall. This shows that the deflection angle of every beam is identical. But the three light pulses from the point $A_{0}^{\prime}$ of the uniform acceleration elevator get to the three different points: $B_{1}^{\prime}, B_{2}^{\prime}$ and $B_{3}^{\prime}$. This shows that the deflection angles of the three beams are different in the elevator reference system. So in the Figure 2 the author draws the three different deflection angles: $\alpha_{1}^{\prime}, \alpha_{2}^{\prime}$ and $\alpha_{3}^{\prime}$. According to formulas (5)-(7), the three deflection angles have the following relationship:

$$
\alpha_{3}^{\prime}>\alpha_{2}^{\prime}>\alpha_{1}^{\prime}
$$

The deflection of light in the gravitational field is not the same as it in the uniform acceleration elevator. So the observer in a closed elevator will know that the elevator is parked on the surface of a planet or in the uniform acceleration movement by observing the light coming from outside, even if he does not look outside. If the light in an elevator is curving and the deflection angle of every beam is coincident or the illumination point on the opposite wall is changeless, it shows that the elevator is parked on the surface of a planet. However, if the light in an elevator is curving, but as time goes on, the deflection angle of light will become bigger and bigger, and the illumination point on the opposite wall is moving down constantly, it shows that the elevator is in the uniform acceleration movement.

According to formulas (2)-(4) and (9), under the condition of $a=10 \mathrm{~m} / \mathrm{s}^{2}$, the au- 
thor figures out the downward distance from the illumination point and the deflection angle of the light when the elevator continues to accelerate $1 \mathrm{~s}, 10 \mathrm{~s}, 100 \mathrm{~s}$ and $600 \mathrm{~s}$. The results are listed in Table 1.

It is not difficult theoretically to use experiments to verify the deflection of light in an acceleration system. Let a small rocket continue a uniform accelerated flight for a period of time. The outside light gets into the rocket from the point $A_{0}^{\prime}$. Through the photosensitive device on the opposite wall we can detect if the illumination point of the light is constantly moving down, and if the deflection angle of the light is becoming bigger and bigger. If the instrument inside the rocket can detect that the light spot is constantly moving down, it shows that the deflections of the light in a uniform accelerated rocket and in a gravitational field are different. The relationship between the acceleration system and the gravitation field is not completely equivalent.

Because the acceleration of the rocket is only $10 \mathrm{~m} / \mathrm{s}^{2}$, so the Space-time curvature effect of the General Theory of Relativity can be ignored; after a sustained acceleration of 600 seconds, the speed of the rocket is only $6 \mathrm{~km} / \mathrm{s}$, so the length contraction effect of the Special theory can be ignored. So when calculating the data in Table 1, the author did not consider the effect of the length contraction and Space-time curvature.

\subsubsection{The Trajectory of the Photon in the Uniform Acceleration Elevator}

In the above example, because the width of the elevator was only $3 \mathrm{~m}$, so when we calculated the deflection angle of the three beams, three curves were replaced by three straight lines. In this section, we will draw out the three curves precisely, thus people can understand that the real trajectories of the photon.

In Figure 3, we used a dashed frame to represent the uniform acceleration elevator, and established a rectangular coordinate system $K$ in the elevator. $A_{0}^{\prime}$ is the origin of the coordinate system, the positive direction of $x$ axis points to right of the elevator, Its coordinate unit is $1 \times 10^{-2} \mathrm{~m}$; the positive direction of $y$ axis points to the above the elevator, Its coordinate unit is $1 \times 10^{-18} \mathrm{~m}$. The time needs for photons to go through a distance of 3 meters is approximately $10 \mathrm{~ns}$. So we take 10 interval points, set $1 \mathrm{~ns}$ as a time interval, and calculate the coordinate values of these three light pulses in each interval, which we needed when drawing out these motion curves of the photons.

In $1 \mathrm{~ns}$ time, the photon pass through $30 \mathrm{~cm}$; in $2 \mathrm{~ns}$ time, the photon pass $60 \mathrm{~cm}$; and so on. So the $x$ coordinate values of the three curves are very easy to calculate, and the calculation of $y$ coordinate value can refer to formula (2)-(4). The whole calculation process is not described in detail, here we only list the calculation results: the data of the first curve is in the Table 2, the data of the second curve is in the Table 3, the data of the third curve is in the Table 4.

Table 1. Downward distance and deflection angle of photon in acceleration system.

\begin{tabular}{ccccc}
\hline Accelerating time & $1 \mathrm{~s}$ & $10 \mathrm{~s}$ & $100 \mathrm{~s}$ & $600 \mathrm{~s}$ \\
Downward distance & $0.1 \mu \mathrm{m}$ & $1 \mu \mathrm{m}$ & $10 \mu \mathrm{m}$ & $60 \mu \mathrm{m}$ \\
Deflection angle & $0.00688^{\prime \prime}$ & $0.0688^{\prime \prime}$ & $0.688^{\prime \prime}$ & $4.13^{\prime \prime}$ \\
\hline
\end{tabular}


Table 2. The data of the first curve.

\begin{tabular}{cccccccccccc}
\hline Time & $t_{0}^{\prime}$ & $t_{01}^{\prime}$ & $t_{02}^{\prime}$ & $t_{03}^{\prime}$ & $t_{04}$ & $t_{05}$ & $t_{06}$ & $t_{07}^{\prime}$ & $t_{08}^{\prime}$ & $t_{09}^{\prime}$ & $t_{1}^{\prime}$ \\
\hline$x$ coordinates & 0 & 30 & 60 & 90 & 120 & 150 & 180 & 210 & 240 & 270 & 300 \\
$y$ coordinates & 0 & -5 & -20 & -45 & -80 & -125 & -180 & -245 & -320 & -405 & -500 \\
\hline
\end{tabular}

Table 3. The data of the second curve.

\begin{tabular}{cccccccccccc}
\hline Time & $t_{1}^{\prime}$ & $t_{11}^{\prime}$ & $t_{12}^{\prime}$ & $t_{13}^{\prime}$ & $t_{14}^{\prime}$ & $t_{15}^{\prime}$ & $t_{16}^{\prime}$ & $t_{17}^{\prime}$ & $t_{18}^{\prime}$ & $t_{19}^{\prime}$ & $t_{2}^{\prime}$ \\
\hline$x$ coordinates & 0 & 30 & 60 & 90 & 120 & 150 & 180 & 210 & 240 & 270 & 300 \\
$y$ coordinates & 0 & -105 & -220 & -345 & -480 & -625 & -780 & -945 & -1120 & -1305 & -1500 \\
\hline
\end{tabular}

Table 4. The data of the third curve.

\begin{tabular}{cccccccccccc}
\hline Time & $t_{2}^{\prime}$ & $t_{21}^{\prime}$ & $t_{22}^{\prime}$ & $t_{23}^{\prime}$ & $t_{24}^{\prime}$ & $t_{25}^{\prime}$ & $t_{26}^{\prime}$ & $t_{27}^{\prime}$ & $t_{28}^{\prime}$ & $t_{29}^{\prime}$ & $t_{3}^{\prime}$ \\
\hline$x$ coordinates & 0 & 30 & 60 & 90 & 120 & 150 & 180 & 210 & 240 & 270 & 300 \\
y coordinates & 0 & -205 & -420 & -645 & -880 & -1125 & -1380 & -1645 & -1920 & -2205 & -2500 \\
\hline
\end{tabular}

In above three tables, the $x$ coordinate unit is $1 \times 10^{-2} \mathrm{~m}$, the $y$ coordinate unit is $1 \times 10^{-18} \mathrm{~m}$. The time internal is $1 \mathrm{~ns}$, namely:

$$
t_{3}^{\prime}-t_{29}^{\prime}=t_{29}^{\prime}-t_{28}^{\prime}=\cdots=t_{2}^{\prime}-t_{19}^{\prime}=t_{19}^{\prime}-t_{18}^{\prime}=\cdots=t_{1}^{\prime}-t_{09}^{\prime}=\cdots=t_{01}^{\prime}-t_{0}^{\prime}=1 \mathrm{~ns}
$$

According to the data in above three tables, we draw out three curves accurately in Figure 3: $\hat{A}_{0}^{\prime} \widehat{B}_{1}^{\prime}, \hat{A}_{0}^{\prime} \widehat{B}_{2}^{\prime}, \hat{A}_{0}^{\prime} \widehat{B}_{3}^{\prime}$. The curve $\hat{A}_{0}^{\prime} \widehat{B}_{1}^{\prime}$ is the trajectory of the first light pulse in uniform acceleration elevator, the curve $\hat{A}_{0}^{\prime} \widehat{B}_{2}^{\prime}$ is the trajectory of the second light pulse, the curve $\hat{A}_{0}^{\prime} \widehat{B}_{3}^{\prime}$ is the trajectory of the third light pulse.

Comparing Figure 1(b) and Figure 3, people could find that each light pulse from the point $A_{0}$ of the stationary elevator always gets to the point $B_{1}$ on the opposite wall in the gravitational field, the path of every beam is identical, and the irradiation point is constant, so the author draw only a curve $\widehat{A}_{0} \widehat{B}_{1}$ and a point of illumination $B_{1}$ in Figure 1(b); but three beams from the point $A_{0}$ of the uniform acceleration elevator, the paths of the three beams are different, so in Figure 3 there were three completely separate curves $\hat{A}_{0}^{\prime} \widehat{B}_{3}^{\prime}, \hat{A}_{0}^{\prime} \widehat{B}_{2}^{\prime}, \hat{A}_{0}^{\prime} \widehat{B}_{1}^{\prime}$ and locations with different illumination points $B_{1}^{\prime}, B_{2}^{\prime}, B_{3}^{\prime}$, as time goes on, the trajectory of the light became more and more curved. Figure 3 once again proved: The deflection of the light in the uniform acceleration elevator is different from the deflection of the light in the gravitational field, there are not completely equivalent relationship between the uniform acceleration elevator and the gravitational field.

\section{Uniform Rotating Disc and Gravitational Field Are Not Completely Equivalent}

Suppose that there is a room on the surface of a planet, and a sagging spring connects a small ball $w$ whose quality is $m$ to the roof. The ball is subjected to two forces: one is the upward spring pull, and the other is the downward gravitational force. The two forces balance, and the ball $w$ is in a stationary status in the room. Suppose again that 
$\mathrm{K}$

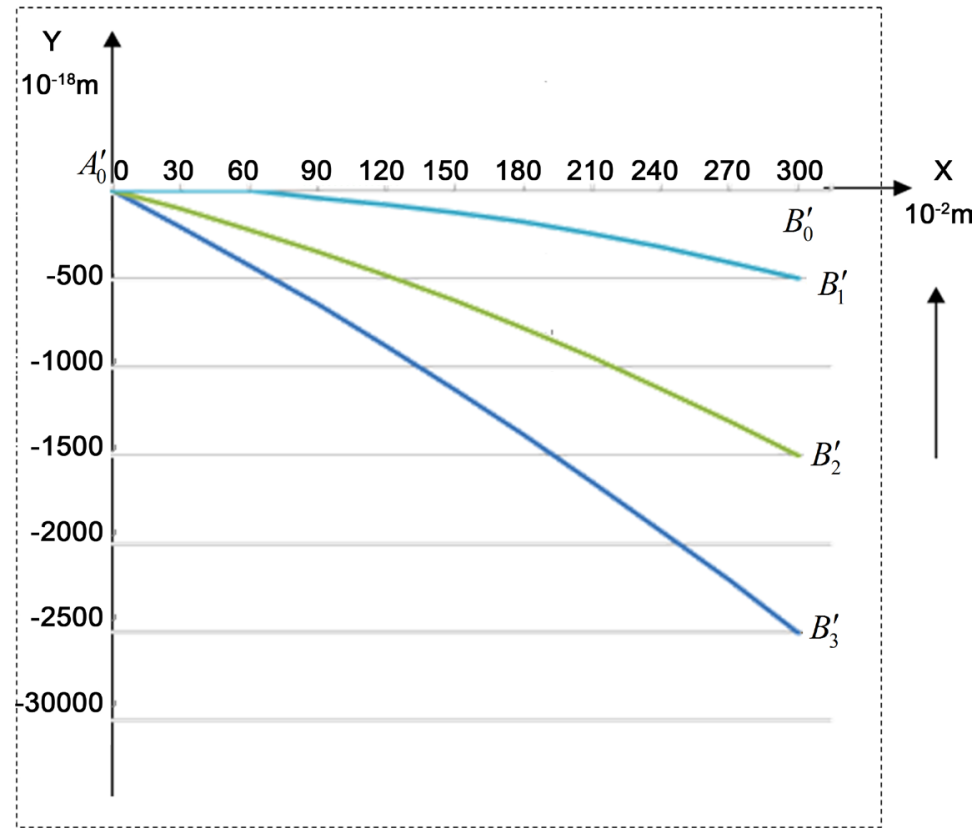

Figure 3. The bending light in the acceleration elevator.

there is a disc in a so-called free space, and a spring connects a small ball $w^{\prime}$ whose quality is $m$ to the center of the disc. When the disc uniform rotation, the ball is also subjected to two forces: One is the spring pull toward the center of the disc, and the other is the centrifugal force opposite to the direction of the spring pull. According to the analysis on the forces of the small ball in the disc reference frame, the spring pull and the centrifugal force balance, and the ball $w^{\prime}$ is in a stationary status in the disc. So Einstein believed that the centrifugal force and the gravitation force are equivalent.

An observer on the disc may think that the disc is stationary, and the small ball is subject to the forces of gravitation and bounce. The two forces balance so that the ball is in a stationary status. Modern physicists generally believe that the uniform rotating disc and the gravitational field are completely equivalent. If the disc is enclosed, the observer on the disc will never be able to use any experiment to distinguish between the centrifugal force and the gravity.

But in fact, it is not the truth. Even if the disc is enclosed, the observer can still distinguish between the centrifugal force and the gravity in free fall experiments of objects. As long as cutting off the spring connecting the roof and the small ball, the observer in the room will see that the ball goes into the free fall status under the action of gravity. The direction of the free fall is consistent with the direction of the gravity. In the whole process of falling, the speed of an object is getting faster and faster, thus the kinetic energy is getting bigger and bigger. But on the uniform rotating disc this scene can't be sawn. At the moment the spring is cut off, the ball will not do the free fall movement in the direction of the centrifugal force, but will fly away instead at the original speed along the direction of tangent line of the circular motion. If the closed disc is large enough, the observer will find that the moving path of the ball is a spiral line and the 
kinetic energy of the flying ball is constant. On the surface of a planet, an object will do free fall movement under gravity action, but on a uniform rotating disc, it is not possible for an object to do the free fall movement under centrifugal force action. According to this, the observer on the disc can distinguish between gravity and centrifugal force. It shows that the uniform rotating disc and the gravitational field are not completely equivalent.

\section{Think about Time Property of Acceleration System and Gravitational Field}

According to the General Theory of Relativity, the intensity of the gravitational field affects the rhythm of the clock. The bigger the gravity, the slower the clock goes. Based on the principle of equivalence, some physicists have come to the conclusion that the more the acceleration is, the more the time expands [2]. But experiments show that acceleration has no effect on the clock rhythm. The most classical experiment to verify the time dilation is to observe the average life span of various kinds of mesons. These experiments can be done in two ways. One is through the observation of cosmic rays from outer space. The mesons of cosmic rays are in a uniform linear motion. The other is to put the meson in a cyclotron and to make it do uniform circular movement. According to the saying: "the more accelerated, the more delay", the meson in cyclotron will have more delays because it has more acceleration movement than the meson in the cosmic ray. But the experiment tells us that whether the meson is making a uniform linear motion or making a uniform circular motion, time dilation is only related to the speed of the clock, and there is no correlation between the acceleration and the time dilation.

Suppose that a meson is in a uniform circular motion in a cyclotron. If the acceleration does affect the rhythm of the clock, as long as the radius of the circular motion of the meson is shortened, under the condition of constant line speed, the average life will be extended. But that never happened. By further analysis of these experiments you will also find that even in the case of $10^{16} \mathrm{~g}$ acceleration, it does not produce any impacts on the clock rate [3]. According to the General Theory of Relativity, the intensity of the gravitational field affects the rhythm of the clock, and according to the results of experiments, the intensity of the acceleration field does not affect the rhythm of the clock. It shows that the acceleration system and the gravitational field is not completely equivalent.

\section{Conclusions}

The author makes some analysis on the equivalence of the acceleration system and the gravitational field, and the results are as follows:

1) According to the author's estimate, the deflections of light in the gravitational field and in the uniform acceleration elevator are not consistent, the uniform acceleration elevator and the gravitational field are not completely equivalent.

2) The object can go into the free fall under the action of gravity, but the centrifugal force on a uniform rotating disc does not have the function. This shows that the 
uniform rotating disc and the gravitational field are not completely equivalent.

3) According to the General Theory of Relativity, the intensity of the gravitational field affects the rhythm of the clock. In a place where the gravity is bigger, the clock goes more slowly. But in the experiment, it was discovered that acceleration had no effect on the clock rhythm. Even when the acceleration reached $10^{16} \mathrm{~g}$, it is still the case. This shows that the time-space relationship between the acceleration system and the gravitational field is not completely equivalent.

4) In the book "Analysis of Relativity Theory" published in 2015, the author carefully thinks about the equivalence relationship between the uniform rotating disc and the uniform acceleration elevator, and gets to the conclusion that there is only a partial equivalence in the acceleration systems and in the gravitational field. There is no completely equivalent relationship [4].

5) The principle of equivalence is an extremely important principle in modern physics. If there are several experiments which can prove that the gravitational field and the accelerating system are not completely equivalent, it will lead to a major change in Physics.

\section{References}

[1] Einstein, A. (1979) Evolution of Physics. Translated by Zhou, Z.W. Shanghai Science and Technology Publishing Company, Shanghai, 163.

[2] Zhu, J.D. (2003) Discussing the Fundament of the Experiment about the Special Relativity. Journal of Shanghai University of Electric Power, No. 3, 57.

[3] Zhu, J.D. (2003) Some Analysis on the Relativistic View of Space-Time. Journal of Shanghai University of Electric Power, No. 1, 50.

[4] Zhu, J.D. (2015) Analysis of Relativity Theory. Dong Hua University Press, CO LTD, 187-195.

Submit or recommend next manuscript to OALib Journal and we will provide best service for you:

- Publication frequency: Monthly

- 9 subject areas of science, technology and medicine

- Fair and rigorous peer-review system

- Fast publication process

- Article promotion in various social networking sites (LinkedIn, Facebook, Twitter, etc.)

- Maximum dissemination of your research work

Submit Your Paper Online: Click Here to Submit

Or Contact service@oalib.com 